\title{
BMJ Open Randomised phase II trial of CAPTEM or FOLFIRI as SEcond-line therapy in NEuroendocrine CArcinomas and exploratory analysis of predictive role of PET/CT imaging and biological markers (SENECA trial): a study protocol
}

To cite: Bongiovanni A, Liverani C, Pusceddu S, et al. Randomised phase II trial of CAPTEM or FOLFIRI as SEcondline therapy in NEuroendocrine CArcinomas and exploratory analysis of predictive role of PET/CT imaging and biological markers (SENECA trial): a study protocol. BMJ Open 2020;10:e034393. doi:10.1136/ bmjopen-2019-034393

- Prepublication history and additional material for this paper are available online. To view these files, please visit the journal online (http://dx.doi org/10.1136/bmjopen-2019034393).

Received 18 September 2019 Revised 14 May 2020 Accepted 09 June 2020

Check for updates

(c) Author(s) (or their employer(s)) 2020. Re-use permitted under CC BY-NC. No commercial re-use. See rights and permissions. Published by BMJ.

For numbered affiliations see end of article.

Correspondence to Dr Alberto Bongiovanni; alberto.bongiovanni@irst.emr.it

\section{ABSTRACT}

Introduction Patients with metastatic or locally advanced, non-resectable, grade 3 poorly differentiated gastroenteropancreatic (GEP) and lung neuroendocrine carcinomas (NECs) are usually treated with in first-line platinum compounds. There is no standard second-line treatment on progression. Accurate biomarkers are needed to facilitate diagnosis and prognostic assessment of patients with NEC.

Methods and analysis The SEcond-line therapy in NEuroendocrine CArcinomas (SENECA) study is a randomised, non-comparative, multicentre phase II trial designed to evaluate the efficacy and safety of folinic acid, 5-fluorouracil and irinotecan (FOLFIRI) or capecitabine plus temozolomide (CAPTEM) regimens after failure of first-line chemotherapy in patients with lung NEC and GEP-NEC. Secondary aims are to correlate the serum miRNA profile and primary mutational status of MEN1, DAXX, ATRX and RB-1 with prognosis and outcome and to investigate the prognostic and predictive role of the Ki-67 score and 18-fluorodeoxyglucose positron emission tomography/computed tomography $\left({ }^{18} \mathrm{~F}-\mathrm{FDG}\right.$ PET/CT) or ${ }^{68} \mathrm{Ga}-\mathrm{PET} / \mathrm{CT}$. The main eligibility criteria are age $\geq 18$ years; metastatic or locally advanced, non-resectable, grade 3 lung or GEP-NECS; progression to first-line platinum-based chemotherapy. A Bryant and Day design taking into account treatment activity and toxicity was used to estimate the sample size. All analyses will be performed separately for each treatment group in the intention-to-treat population. $A$ total of 112 patients (56/arm) will be randomly assigned (1:1) to receive FOLFIRI every 14 days or CAPTEM every 28 days until disease progression or unacceptable toxicity or for a maximum of 6 months. Patients undergo testing for specific biomarkers in primary tumour tissue and for miRNA in blood samples. MiRNA profiling will be performed in the first 20 patients who agree to participate in the biological substudy. Ethics and dissemination The SENECA trial, supported by Istituto Scientifico Romagnolo per lo Studio e la Cura
Strengths and limitations of the study

- The SEcond-line therapy in NEuroendocrine CArcinomas (SENECA) trial randomises patients to receive two different treatments, folinic acid, 5-fluorouracil and irinotecan or capecitabine plus temozolomide, providing important information on the activity of both combinations in different neuroendocrine carcinoma (NEC) subtypes (neuroendocrine tumour grade (G) 3 and NEC G3).

- The SENECA trial analyses the role of miRNAs and other biological markers as prognostic and predictive factors. A further aim is to assess ${ }^{68} \mathrm{Ga}-\mathrm{PET} / \mathrm{CT}$ as a tool to improve current histological classification.

- The rarity of the disease and patient prognosis. However, the involvement of several Italian centres will hopefully help to overcome this problem.

- Poor prognosis of patients with NEC. Patients progressing on platinum chemotherapy usually have rapid deterioration of clinical conditions.

dei Tumori (IRST), was authorised by the locals Ethics Committee and the Italian Medicines Agency (AIFA). Results will be widely disseminated via peer-reviewed manuscripts, conference presentations and reports to relevant authorities. The study is currently open in Italy.

Trail registration number NCT03387592; Pre-results. EudraCT-2016-000767-17.

Protocol version Clinical Study Protocol Version 1, 7 November 2016.

\section{INTRODUCTION}

Poorly differentiated neuroendocrine carcinomas (NECs) are very rare malignancies, representing only $5 \%-10 \%$ of 
neuroendocrine neoplasias (NENs). ${ }^{1-3}$ At the time of diagnosis, patients are generally in poor conditions due to aggressive and diffuse disease. These tumours are characterised by aggressive histological features (high Ki-67 index, extensive necrosis and nuclear atypia) and are classified as grade (G) 3 NECs according to the 2010 WHO classification. ${ }^{4}$ The 2017 WHO classification recognised a further group called G3 neuroendocrine tumours (NETs) as having intermediate features between NETs and NECs. ${ }^{5}$

An etoposide-platinum combination is the gold standard for the treatment of G3 NECs, several studies published in the 1990s reporting substantial antitumour activity and high response rates $(41 \%-67 \%) .{ }^{6}$ However, prognosis is generally poor with a median progressionfree survival (PFS) of 9 months and a median overall survival (OS) of 15-19 months. When progression occurs after first-line chemotherapy, the disease is usually very aggressive and patients succumb rapidly. ${ }^{7}$

Given the rarity of this disease, prospective clinical data are lacking and treatment recommendations are essentially expert-based opinions. Two phase II studies investigating the second-line treatment of gastroenteropancreatic (GEP)-NECs are currently registered at ClinicalTrials.gov: one evaluating the safety and tolerability of everolimus in 40 patients with G3 NEC/NET or G1/ G2 NET switching to G3 NEN (National Clinical Trial identifier NCT02113800) and the other investigating the efficacy of avelumab (NCT03147404). A French study focusing on the identification of predictive molecular markers of response to sunitinib in poorly differentiated digestive NETs (NCT01215578) has now closed recruitment and results are eagerly awaited. Another French multicentre prospective phase II trial is currently ongoing to investigate the efficacy of the bevacizumab-folinic acid, 5-fluorouracil and irinotecan (FOLFIRI) combination after progression on a platinum/etoposide combination. ${ }^{7}$

Different second-line chemotherapy combinations have been evaluated but shown poor results. ${ }^{8-10}$ In a monocentre retrospective clinical trial, Hentic et al hypothesised the potential efficacy of FOLFIRI as second-line chemotherapy in 19 patients with G3 extra-pulmonary NECs. ${ }^{11}$ An objective response rate was obtained in $31 \%$ of patients, with a disease control rate (DCR) of $62 \%$. Median PFS and OS were 4 and 18 months, respectively.

In another retrospective study, a $71 \%$ DCR was obtained with temozolomide-based chemotherapy in 25 patients with metastatic poorly differentiated endocrine carcinoma of different sites and atypical bronchial carcinoid with $\mathrm{Ki}-67>20 \%$. Small cell lung carcinoma and large cell lung carcinoma were excluded. A PFS of 12 months (95\% CI 5.5 to 24) and OS of 22 months (95\% CI 12 to $31)$ were reported in patients who responded to treatment or showed stable disease (SD), whereas OS was only 8 months (95\% CI 0 to 8 ) in non-responders. The authors observed a higher response rate in patients with Ki-67 $\leq 60 \%$. There were also more responders in the group with high uptake in somatostatin receptor scintigraphy and in those with positive staining for chromogranin A. Both factors are often associated with more highly differentiated tumours. ${ }^{12}$

Literature data on lung NECs in progression after firstline chemotherapy are based on small patient series. ${ }^{13}$ Moreover, there is increasing evidence of some discrepancies in the current grading of NECs, highlighting the need for more accurate biomarkers. ${ }^{45}$ Recent research has shown that NECs may, in fact, comprise two distinct subgroups with different pathogenesis, that is, a highly proliferative group derived from well-differentiated NETs and characterised by mutations in MEN1, DAXX and ATRX, and a poorly differentiated group derived from neuroendocrine-differentiated adenocarcinomas and characterised by a mutation in RB1. Both subgroups display a distinct prognosis and different sensitivity to chemotherapy. ${ }^{14-16}$ Micro(mi)RNAs are a class of small, non-coding, highly conserved single-stranded RNAs involved in the post-transcriptional regulation of cell proliferation, differentiation, survival and apoptosis. ${ }^{17}$ They are often associated with resistance to therapy. ${ }^{18} 19$ While miRNAs are known to show a specific expression pattern in NETs, ${ }^{20}$ little is known about differential miRNA profiles in patients with NEC. At present, no data are available on the deregulation of specific miRNAs in this setting.

In a study recently published by our group on patients with GEP-NEC undergoing first-line platinum-based chemotherapy, median PFS was 19.3 months and 6.3 months $(\mathrm{p}<0.01)$ in patients with $\mathrm{Ki}-67$ value between $20 \%$ and $50 \%$ or $>50 \%$, respectively. ${ }^{19}$ Median (m) OS was 8.1 months in the latter group but was not reached in the former group $(\mathrm{p}=0.039)$. Patients with a positive ${ }^{68} \mathrm{Ga}-\mathrm{PET} / \mathrm{CT}$ had a higher 18 month OS rate than those with a negative scan $(75 \%$ vs $34.3 \%$, respectively), but the difference was not statistically significant $(p=0.06)$. Our data highlighted that ${ }^{68} \mathrm{Ga}-\mathrm{PET} / \mathrm{CT}$ positivity may be a discriminating factor ${ }^{16}{ }^{21}$ in predicting prognosis, especially in the metastatic setting where histological material is not always available for evaluation. Furthermore, 18-fludeoxyglucose $\left({ }^{18} \mathrm{FDG}\right)$-PET/CT may be useful to discriminate between patients with different prognosis. ${ }^{22}$

Given the above premises, we decided to investigate the efficacy and safety of second-line FOLFIRI or capecitabine plus temozolomide (CAPTEM) in patients with GEP and lung NECs in progression after first-line platinum-based treatment. We also aimed to study the serum miRNA profile in relation to the primary mutational status of MEN1, DAXX, ATRX and RB-1, patient prognosis and response to therapy, and to assess the prognostic and predictive role of ${ }^{18} \mathrm{FDG}$-PET/CT, ${ }^{68} \mathrm{Ga}-\mathrm{PET} /$ CT and Ki-67 score.

\section{METHODS AND ANALYSIS Study design}

The SEcond-line therapy in NEuroendocrine CArcinomas (SENECA) study is a multicentre randomised 


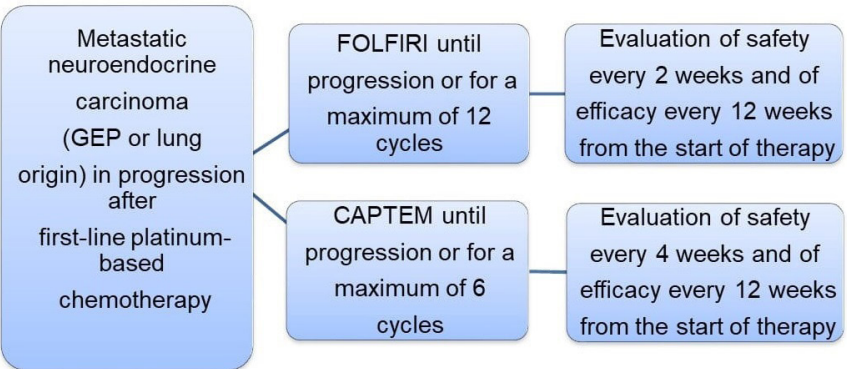

Figure 1 SEcond-line therapy in NEuroendocrine CArcinomas (SENECA) study design. CAPTEM, capecitabine plus temozolomide; FOLFIRI, folinic acid, 5-fluorouracil and irinotecan; GEP, gastroenteropancreatic.

non-comparative phase II study (figure 1). Patients with metastatic NECs of different origin (lung or GEP) in progression after first-line treatment are randomised to receive FOLFIRI every 14 days for a maximum of 12 cycles or until progression or unacceptable toxicity, or CAPTEM every 28 days for a maximum of 6 cycles or until progression or unacceptable toxicity.

The treatment arms are as follows:

\section{FOLFIRI regimen}

- Irinotecan $180 \mathrm{mg} / \mathrm{m}^{2}$, given as a $60 \mathrm{~min}$ intravenous infusion on day 1 every 2 weeks followed by

- Leucovorin $200 \mathrm{mg} / \mathrm{m}^{2}$, given as a 2 hours intravenous infusion on day 1 every 2 weeks followed by

- 5-fluorouracil (5-FU) $400 \mathrm{mg} / \mathrm{m}^{2}$ given as bolus, and then 5 -FU $2400 \mathrm{mg} / \mathrm{m}^{2}$ given as a 48 hours continuous infusion on day 1 , every 2 weeks, until progression or for a maximum of 12 cycles.

\section{CAPTEM regimen}

Capecitabine $750 \mathrm{mg} / \mathrm{m}^{2}$ two times a day on days 1-14 in combination with temozolomide $200 \mathrm{mg} / \mathrm{m}^{2}$ daily on days 10-14, every 4 weeks, until progression or for a maximum of 6 cycles.

The study includes patients aged $\geq 18$ years with a histological diagnosis of G3 NEC (GEP-NEC and lung NEC) according to the 2010 and 2015 GEP and lung NEN WHO classifications, respectively, Ki-67 $>20 \%$ and measurable disease according to Response Evaluation Criteria In Solid Tumors (RECIST) 1.1 criteria. All patients must have an Eastern Cooperative Oncology Group performance status $\leq 2$ with a life expectancy $>3$ months and must have already undergone first-line treatment for metastatic disease with platinum-based chemotherapy (cisplatin/carboplatin and etoposide, FOLFOX4 or CAPOX). Adequate haematological, liver and renal function is required and effective contraceptive methods must be used by female patients of childbearing age. Written informed consent is obtained from all patients to take part in the study. Exclusion criteria are as follows: metastatic NECs previously treated with an irinotecan or temozolomide regimen, known hypersensitivity to 5-FU/capecitabine, calcium levofolinate, irinotecan or their recipients. All acute toxic effects of any prior therapy (including surgery, radiation therapy and chemotherapy) must have resolved to grade $\leq 1$ according to National Cancer Institute Common Terminology Criteria for Adverse Events version 4.03 (CTCAE). Patients taking part in another clinical trial with any investigational agent $<30$ days prior to study screening or with a history of allergic reactions attributable to compounds of similar chemical or biological composition are excluded. Patients who have undergone chemotherapy or radiotherapy $<4$ weeks ( 6 weeks for nitrosoureas or mitomycin C) prior to entering the study have not recovered from adverse events caused by agents administered $>4$ weeks earlier, or have known brain metastases are not eligible for the study. Patients with other malignancies with a disease-free interval of $<5$ years (with the exception of non-melanoma skin cancer or low-grade superficial bladder cancer) are excluded, as are those with any severe and/or uncontrolled medical condition or other condition that could affect their participation in the study such as:

- Unstable angina pectoris, symptomatic congestive heart failure, myocardial infarction $<6$ months before the start of the study, serious uncontrolled cardiac arrhythmia or any other clinically significant cardiac disease.

- Severely impaired lung function (spirometry and diffusing capacity of the Lung for carbon monoxide (DLCO) $50 \%$ of the normal predicted value and/or oxygen saturation $\leq 88 \%$ at rest, in room air).

- Uncontrolled diabetes as defined by fasting serum glucose $>1.5 \times$ upper limit of normal.

- Any active (acute or chronic) or uncontrolled infections/disorders.

Tumour evaluation by anatomic imaging (multiphase CT and/or MRI) includes chest, abdomen, pelvis and any additional known sites of disease. These tests are performed at baseline, every 3 months during treatment as per national regulatory agency indications, and after the end of treatment in non-progressing patients until progression. It is recommended that ${ }^{68} \mathrm{Ga}-\mathrm{PET} / \mathrm{CT}$ and ${ }^{18}$ FDG-PET/CT scans be performed at baseline or a maximum of 90 days before study enrolment. An European Organization for the Research and Treatment of Cancer Quality of Life Questionnaire (EORTC QLQ-C30) is administered at baseline and every 3 months thereafter during the treatment period.

\section{Study endpoints}

The primary endpoint of the study is the DCR of each treatment, defined as the percentage of patients who have achieved complete or partial response or SD for $\geq 12$ weeks from the start of therapy. DCR will be evaluated using the new international criteria proposed by the RECIST version 1.1. Acute and late toxicity will be assessed by CTCAE version 4.03, the latter defined as toxicity occurring at least 30 days after the end of the last treatment cycle. Secondary endpoints are OS, calculated from the start of treatment to death from any cause, and PFS, calculated from the start of treatment to the date 
of the first documented evidence of disease progression or of death from any cause. All the analyses will be performed in the intention-to-treat population. Patients without events at the time of analysis will be censored at their last-known-alive date for OS and at their last date of tumour evaluation for PFS. A further secondary endpoint is the evaluation of quality of life using the European Organization for the Research and Treatment of Cancer Quality of Life Questionnaire (EORTC QLQ-C30). When data are available, the impact of baseline ${ }^{68} \mathrm{Ga}-\mathrm{PET} / \mathrm{CT}$ and ${ }^{18}$ FDG-PET/CT on PFS will be analysed with exploratory intent. After signing the informed consent for biomarker assessment, patients will undergo evaluation of the mutation status of MEN1, DAXX, ATRX and RB-1 in primary tumour tissue and of miRNA in blood samples. Assessment of the miRNA profile will be performed on the first 20 patients who agree to participate in the biological part of the study.

\section{Ethical considerations}

The present clinical trial, supported by Istituto Scientifico Romagnolo per lo Studio e la Cura dei Tumori (IRST), was authorised by the local Ethics Committee and by the Italian Medicines Agency (AIFA). The request for EudraCT registration (mandatory for studies in Europe) was send to AIFA in December 2016 and we received a EudraCT number (EudraCT 2016-000767-17). However, technical problems at AIFA resulted in some clinical trials, including ours, being uploaded onto the EudraCT website after enrolment of the first patients.

The study is also registered on the ClinicalTrials.gov website (NCT03387592). The study complies with the ethical standards laid down in the 1964 Declaration of Helsinki and the principles of Good Clinical Practice guidelines (including written informed consent).

\section{Patient and public involvement}

This research work was performed without patient involvement in the study design, execution or outcome measures.

\section{Statistical methods}

The Bryant and Day design is used to estimate a sample size that takes into account both treatment activity and toxicity. Although randomisation is used to allocate patients to the two arms, no formal statistical comparisons between treatment regimens are planned. The purpose of randomisation is to reduce bias due to patient assignment to a specific treatment arm. The hypothesis for the control arm is based on literature data. ${ }^{623}$

An $\alpha$ level of 0.10 (both for toxicity and DCR) and a power of $90 \%$ have been adopted. A DCR rate $\geq 60 \%$ and a relevant toxicity rate $\leq 20 \%$ are considered acceptable rates, while a DCR rate $\leq 40 \%$ and a relevant toxicity rate $\geq 40 \%$ are considered inacceptable rates. Given these hypotheses, the first step of the study will require 25 patients. If $\geq 10$ patients with a DCR are observed and $\geq 15$ patients do not have significant toxicity, the study will enrol patients in the next step. A total of 53 patients will be enrolled. If $\geq 25$ patients with DCR and $\geq 36$ patients without any relevant toxicity are observed, treatment will be considered active and not toxic. This design is used for each treatment scheme and all analyses will be performed separately. If one of the schemes does not obtain the expected proportions of the first step, the arm will be closed and patients will be enrolled in the other arm until the target is reached; if the expected proportions are not reached in any arm, the study will be prematurely closed. If no premature stop occurs, a total of 106 evaluable patients are needed (53 patients in each arm). Taking into account a $5 \%$ dropout rate, 56 patients must be enrolled in each arm (total 112 patients). G3-4 gastrointestinal toxicity, G4 thrombocytopoenia, prolonged G3-G4 neutropoenia ( $>7$ days) and drug-related hospitalisations are considered relevant toxicity. The stratification factors of this study are $\mathrm{Ki}-67(21 \%-55 \%$ vs $>55 \%)$ and site of primary tumour (lung vs GEP). A subgroup analysis of the efficacy of both treatments according to these stratification factors has been planned.

Complete response, partial response or SD for at least 12 weeks will be considered as the DCR. The proportion of patients in this category will be determined and $95 \%$ CIs for the DCR will be calculated. OS and PFS will be estimated using the Kaplan-Meier method (two-sided 95\% CIs). ${ }^{23}$ Appropriate statistical analyses will be performed on the basis of the data available to compare QLQ-C30 scores between baseline and subsequent follow-up visits.

When data are available, the impact of ${ }^{68} \mathrm{Ga}$-PET/CT result on PFS will be analysed with exploratory intent. The Shapiro-Wilk test will be used to determine the normality distribution of each clinical, demographic and biological biomarker. ${ }^{24}$ In the event of a non-normal distribution, non-parametric statistics will be used to analyse the relationship between the serum levels of each marker, considered as continuous variables, and response to treatment. In the event of normal biomarker distribution, a parametric test will be used. All endpoints will be analysed separately for each treatment group.

\section{DISCUSSION}

There is still no truly effective second-line chemotherapy for NEC. Overall prognosis of patients is poor, with an OS of 5 months in the metastatic setting according to Surveillance, Epidemiology, and End Results data. ${ }^{25}$ Only $5 \%$ of all patients are long-term survivors. There is also a marked lack of prognostic and predictive factors. ${ }^{5}$

Three phase II studies registered at ClinicalTrials. gov are currently investigating second-line treatment of GEP-NECs: the first focusing on FOLFIRI and bevacizumab (NCT02820857), the second on everolimus (NCT02113800) and the third on avelumab (NCT03147404). Some abstracts were presented at European Society for Medical Oncology 2018 and American Society of Clinical Oncology (ASCO) 2019 on the use of immunotherapy in GEP-NECs, all showing inconclusive 
results. The SENECA study uses a promising approach to the treatment of patients with metastatic NECs. First, both the activity and safety of two regimens are assessed in the same setting with a sizeable patient population (56 patients/arm). In addition, patients are stratified according to Ki-67 index and morphology to investigate the role of each treatment combination in both poorly differentiated and well-differentiated NECs. Another aim of this study is to integrate both biological and metabolic imaging data in an effort to improve the current GEPNEC classification.

The duration of treatments in the metastatic setting is a dilemma in NENs and especially in NECs. Given the lack of evidence-based recommendations on treatment duration of second-line chemotherapy in NECs, we decided to opt for a fixed duration of treatments to avoid unnecessary exposure to cytotoxic agents and consequent bone marrow reserve depletion. ${ }^{26}$

In conclusion, there are still no Food and Drug Administration/European Medicines Agency-approved secondline therapeutic options for patients with metastatic NECs, and the SENECA trial could represent a step forward in finding novel therapies to prolong survival and maintain quality of life. Moreover, the integration of biological and imaging data could lead to a better understanding of the natural history of the disease and help to identify potential responders.

\section{Confidentiality}

This study will be conducted in full conformity with The International Council for Harmonisation (ICH) of Technical Requirements for Pharmaceuticals for Human Use Guidelines for Good Clinical Practice, Directive 2001/20/ EEC of the European Parliament and other relevant current local legislation. Participants will be allocated a unique identification (ID) number at entry. The master list linking participant personal information and ID number will be maintained in a separate locked cabinet and password-protected hard drive. Data will be analysed by ID number only. Patient files and other source data will for be kept a maximum of 15 years.

\section{Ethics and dissemination}

The SENECA trial, supported by IRST, involves several Italian centres and was authorised by the local Ethics Committees of the centres taking part and by the Italian Medicines Agency (AIFA) (see list of all centres in the online supplementary table 1). After completing the study, all data, including beneficial and adverse events, of the trial will be communicated at scientific meetings and published in indexed peer-reviewed journals. If shown to be effective, the therapy programme will be made available to the general public in an appropriate manner.

\section{Author affiliations}

${ }^{1}$ Osteoncology and Rare Tumors Center, Istituto Scientifico Romagnolo per lo Studio e la Cura dei Tumori IRCCS, Meldola, Italy

${ }^{2}$ Department of Medical Oncology, Istituto Nazionale per lo Studio e la Cura dei Tumori, Milano, Lombardia, Italy
${ }^{3}$ Oncology Unit, Ospedale Vito Fazzi, Lecce, Puglia, Italy

${ }^{4}$ Oncology Unit, Ospedale di Bolzano, Bolzano, Trentino-Alto Adige, Italy

${ }^{5}$ Oncology Unit, Ospedale degli Infermi di Faenza, Faenza, Emilia-Romagna, Italy

${ }^{6}$ Department of Medical Oncology, Campus Bio-Medico University, Roma, Lazio, Italy

${ }^{7}$ Department of Oncology and Hematology, Azienda Ospedaliero-Universitaria di

Modena, Modena, Emilia-Romagna, Italy

${ }^{8}$ Medical Oncology Unit, Azienda Ospedaliero-Universitaria di Parma, Parma, EmiliaRomagna, Italy

${ }^{9}$ Oncology Clinic, University Hospital of Ancona Umberto I G M Lancisi G Salesi, Ancona, Marche, Italy

${ }^{10}$ Department of Oncology, Istituto Nazionale di Ricovero e Cura a Carattere Scientifico Saverio de Bellis, Castellana Grotte, Puglia, Italy

${ }^{11}$ Department of Clinical and Experimental Oncology, Istituto Oncologico Veneto

Istituto di Ricovero e Cura a Carattere Scientifico, Padova, Veneto, Italy

${ }^{12}$ Internal Medicine and Medical Oncology, Santa Chiara Hospital, Pisa, Toscana, Italy

${ }^{13}$ Unit of Biostatistics and Clinical Trials, Istituto Scientifico Romagnolo per lo Studio e la Cura dei Tumori IRCCS, Meldola, Italy

${ }^{14}$ Nuclear Medicine Unit, Istituto Scientifico Romagnolo per lo Studio e la Cura dei Tumori IRCCS, Meldola, Italy

Acknowledgements The authors thank Gráinne Tierney and Cristiano Verna for editorial assistance.

Collaborators SENECA study Team investigators: Mauro Cives, Davide Campana, Nicola Silvestris, Angela Buonadonna, Giuseppe Badalamenti, Maria Pia Brizzi, Alfredo Berruti, Francesca Spada, Sara Cingarlini, Lorenzo Antonuzzo, Davide Pastorelli.

Contributors $A B, C L$ and TI designed the study and drafted the article. AB was responsible for data acquisition. SP, SL, GDM, SS and ST provided methodological support and designed a clinical information data extraction method for the protocol. FF performed the statistical analysis. DS, FG, FP, RB, IL, FB and SR revised the paper for important intellectual content. All authors read and approved the present version of the manuscript for submission.

Funding The authors have not declared a specific grant for this research from any funding agency in the public, commercial or not-for-profit sectors.

Competing interests None declared.

Patient and public involvement Patients and/or the public were not involved in the design, or conduct, or reporting, or dissemination plans of this research.

Patient consent for publication Not required.

Provenance and peer review Not commissioned; externally peer reviewed.

Open access This is an open access article distributed in accordance with the Creative Commons Attribution Non Commercial (CC BY-NC 4.0) license, which permits others to distribute, remix, adapt, build upon this work non-commercially, and license their derivative works on different terms, provided the original work is properly cited, appropriate credit is given, any changes made indicated, and the use is non-commercial. See: http://creativecommons.org/licenses/by-nc/4.0/.

ORCID iD

Alberto Bongiovanni http://orcid.org/0000-0002-3845-4687

\section{REFERENCES}

1 Kulke $\mathrm{MH}$, Shah $\mathrm{MH}$, Benson $\mathrm{AB}$, et al. Neuroendocrine tumors, version 1.2015. J Natl Compr Canc Netw 2015;13:78-108.

2 Modlin IM, Moss SF, Chung DC, et al. Priorities for improving the management of gastroenteropancreatic neuroendocrine tumors. $J$ Natl Cancer Inst 2008;100:1282-9.

3 Yao JC, Hassan M, Phan A, et al. One hundred years after "carcinoid": epidemiology of and prognostic factors for neuroendocrine tumors in 35,825 cases in the United States. JCO 2008;26:3063-72.

4 Fraenkel M, Kim M, Faggiano A, et al. Incidence of gastroenteropancreatic neuroendocrine tumours: a systematic review of the literature. Endocr Relat Cancer 2014;21:R153-63.

5 Lloyd RV, Osamura RY, KIöppel G, et al. WHO classification of tumours of endocrine organs. 4th edn. Lyon: International Agency for Research on Cancer (IARC), 2017.

6 Sorbye $\mathrm{H}$, Welin S, Langer SW, et al. Predictive and prognostic factors for treatment and survival in 305 patients with advanced 
gastrointestinal neuroendocrine carcinoma (WHO G3): the NORDIC NEC study. Ann Oncol 2013;24:152-60.

7 Heetfeld M, Chougnet CN, Olsen IH, et al. Characteristics and treatment of patients with G3 gastroenteropancreatic neuroendocrine neoplasms. Endocr Relat Cancer 2015;22:657-64.

8 Walter T, Malka D, Hentic O, et al. Evaluating bevacizumab in combination with FOLFIRI after the failure of platinumetoposide regimen in patients with advanced poorly differentiated neuroendocrine carcinoma: the PRODIGE 41-BEVANEC randomized phase II study. Dig Liver Dis 2018;50:195-8.

9 Hadoux J, Malka D, Planchard D, et al. Post-first-line FOLFOX chemotherapy for grade 3 neuroendocrine carcinoma. Endocr Relat Cancer 2015;22:289-98.

10 Olsen IH, Sørensen JB, Federspiel B, et al. Temozolomide as second or third line treatment of patients with neuroendocrine carcinomas. Sci World J (2012) 2012;2012:1-4.

11 Hentic O, Hammel P, Couvelard A, et al. FOLFIRI regimen: an effective second-line chemotherapy after failure of etoposideplatinum combination in patients with neuroendocrine carcinomas grade 3. Endocr Relat Cancer 2012;19:751-7.

12 Welin S, Sorbye H, Sebjornsen S, et al. Clinical effect of temozolomide-based chemotherapy in poorly differentiated endocrine carcinoma after progression on first-line chemotherapy. Cancer 2011;117:4617-22.

13 Noda K, Nishiwaki Y, Kawahara M, et al. Irinotecan plus cisplatin compared with etoposide plus cisplatin for extensive small-cell lung cancer. N Engl J Med 2002;346:85-91.

14 Derks JL, Leblay N, Thunnissen E, et al. Molecular subtypes of pulmonary large-cell neuroendocrine carcinoma predict chemotherapy treatment outcome. Clin Cancer Res 2018;24:33-42.

15 Tang LH, Untch BR, Reidy DL, et al. Well-differentiated neuroendocrine tumors with a morphologically apparent highgrade component: a pathway distinct from poorly differentiated neuroendocrine carcinomas. Clin Cancer Res 2016;22:1011-7.
16 Liverani C, Bongiovanni A, Mercatali L, et al. Grading of neuroendocrine carcinomas: correlation of $68 \mathrm{Ga}-\mathrm{PET} / \mathrm{CT}$ scan with tissue biomarkers. Dis Markers 2018;2018:1-8.

17 Grolmusz VK, Kövesdi A, Borks K, et al. Prognostic relevance of proliferation-related miRNAs in pancreatic neuroendocrine neoplasms. Eur J Endocrinol 2018;179:219-28.

18 Gill P, Kim E, Chua TC, et al. MiRNA-3653 is a potential tissue biomarker for increased metastatic risk in pancreatic neuroendocrine tumours. Endocr Pathol 2019;30:128-33.

19 Panarelli N, Tyryshkin K, Wong JJM, et al. Evaluating gastroenteropancreatic neuroendocrine tumors through microRNA sequencing. Endocr Relat Cancer 2019;26:47-57.

20 Malczewska A, Kidd M, Matar S, et al. A comprehensive assessment of the role of miRNAs as biomarkers in gastroenteropancreatic neuroendocrine tumors. Neuroendocrinology 2018;107:73-90.

21 Bongiovanni A, Riva N, Ricci M, et al. First-line chemotherapy in patients with metastatic gastroenteropancreatic neuroendocrine carcinoma. Onco Targets Ther 2015;8:3613-9.

22 Sansovini M, Severi S, lanniello A, et al. Long-term follow-up and role of FDG PET in advanced pancreatic neuroendocrine patients treated with 177Lu-D OTATATE. Eur J Nucl Med Mol Imaging 2017;44:490-9.

23 Perren A, Couvelard A, Scoazec J-Y, et al. ENETS consensus guidelines for the Standards of care in neuroendocrine tumors: pathology: diagnosis and prognostic stratification. Neuroendocrinology 2017;105:196-200.

24 Sorbye H, Köhne C-H, Sargent DJ, et al. Patient characteristics and stratification in medical treatment studies for metastatic colorectal cancer: a proposal for standardization of patient characteristic reporting and stratification. Ann Oncol 2007;18:1666-72.

25 Kaplan EL, Meier P. Nonparametric estimation from incomplete observations. J Am Stat Assoc 1958;53:457-81.

26 Strosberg JR, Fine RL, Choi J, et al. First-line chemotherapy with capecitabine and temozolomide in patients with metastatic pancreatic endocrine carcinomas. Cancer 2011;117:268-75. 